Case Report

\title{
Abnormal self-location and vestibular vertigo in a patient with right frontal lobe epilepsy
}

\author{
Christophe Lopez ${ }^{\mathrm{a}}$, Lukas Heydrich ${ }^{\mathrm{a}}$, Margitta Seeck ${ }^{\mathrm{b}}$, Olaf Blanke ${ }^{\mathrm{a}, \mathrm{b}, *}$ \\ ${ }^{a}$ Laboratory of Cognitive Neuroscience, Brain Mind Institute, Ecole Polytechnique Fédérale de Lausanne, Lausanne, Switzerland \\ ${ }^{\mathrm{b}}$ Department of Neurology, EEG and Epilepsy Unit, University Hospital, Geneva, Switzerland
}

\section{A R T I C L E I N F O}

\section{Article history:}

Received 2 December 2009

Revised 16 December 2009

Accepted 18 December 2009

Available online 25 January 2010

\section{Keywords:}

Embodiment

Vestibular system

Frontal cortex

Out-of-body experience

First-person perspective

Multisensory integration

\begin{abstract}
A B S T R A C T
We describe the case of a 33-year-old man with complex partial seizures characterized by the feeling of being projected outside his body, including dissociation of "mind and self from body" (disembodiment), followed by vestibular vertigo due to right frontal lobe epilepsy caused by an oligodendroglioma. We distinguish the patient's ictal symptoms with respect to autoscopic phenomena (out-of-body experience, heautoscopy, autoscopic hallucinations) and vestibular phenomena of epileptic origin, and we discuss their neural origin with respect to vestibular and multisensory cortical mechanisms of bodily self-consciousness in temporoparietal and frontal cortex.
\end{abstract}

(c) 2009 Elsevier Inc. All rights reserved.

\section{Introduction}

Self-location can be defined as the volume in space where humans localize their center of conscious experience. Although normal self-location is experienced as being within the physical boundaries of one's body ("embodiment"), this spatial unity between the self and the body may break down during paroxysmal neurological conditions such as out-of-body experiences (OBEs) and heautoscopy [1-3]. Patients with OBEs for example experience the world from a location and perspective outside their physical body (abnormal first-person perspective [1PP]) and present the most striking case of disturbed self-location.

OBEs have been reported in epilepsy, migraine, neoplasia, infarction, schizophrenia, depression, and depersonalization [1,35]. Abnormal self-location (or "disembodiment") and 1PP during OBEs of epileptic origin has been reported in patients with complex partial seizures affecting the temporoparietal junction (TPJ) $[1,3,5]$ and have been hypothesized to be caused by a failure to integrate multisensory (visual, somatosensory, vestibular) signals at the TPJ, resulting in a breakdown of the spatial unity between the self and the body $[3,6]$.

\footnotetext{
* Corresponding author. Address: Laboratory of Cognitive Neuroscience, Brain Mind Institute, Ecole Polytechnique Fédérale de Lausanne (EPFL), Swiss Federal Institute of Technology, Station 19, 1015 Lausanne, Switzerland. Fax: +41 (0)21 693 9625.

E-mail addresses: christophe.lopez@epfl.ch (C. Lopez), olaf.blanke@epfl.ch (O. Blanke).
}

Although the contribution of visual and somatosensory cues to self-location is largely attested by clinical and experimental data, less is known about the contribution of vestibular cues. Here, we describe a patient with right frontal lobe epilepsy caused by an oligodendroglioma whose seizures were characterized by abnormal self-location and vestibular sensations of vertigo, but without other experiential changes normally associated with OBEs.

\section{Case report}

The patient, a 33-year-old left-handed man (computer technician), was initially referred to our hospital because of a single episode of a generalized tonic-clonic seizure. Although his medical history prior to admission had been unremarkable, clinical and neuroradiological evaluation revealed a right frontal oligodendroglioma grade II (see below), which was surgically resected. From 6 months after surgery the patient complained about the repetitive experience of extracorporeal self-location, or disembodiment, associated with sensations of vertigo. This was characterized by "the feeling as if being projected out of his body" and a dissociation of mind and body for a few seconds. There were no associated visual changes, notably no changes in $1 \mathrm{PP}$, and no autoscopy (as is classically reported in OBEs, e.g., [3]). This experience was always followed by strong vestibular sensations with rotatory vertigo and loss of balance and occurred several times per day. On other occasions the patient reported isolated sensations of rotatory ver- 
tigo (without disembodiment) followed by nausea, vomiting, and an altered sense of touch affecting his whole body ("as if everything was cold") lasting about $1 \mathrm{~min}$. These episodes were followed by postictal ageusia and occurred five times in total. Three shorter episodes ( $\sim 1 \mathrm{~min}$ ) characterized by accelerated thinking, ultrarapid calculation, heightened consciousness, and euphoria were also noted, but were never associated with disembodiment, vestibular, or somatosensory sensations. These experiences started 6 months after surgery and increased in frequency.

Postoperative MRI revealed a cortical and subcortical nodular lesion enhancing with contrast in proximity to the resection in the right frontal lobe (Fig. 1), indicating a relapse of the oligodendroglioma (grade III). These findings were confirmed by EEG (see below), PET, and single-photon-emission computed tomography (SPECT), which showed two nodular hypermetabolic and hyperperfused zones posterior and adjacent to the resection. Interictal EEG revealed an epileptic focus with continuous theta and delta slowing over the right frontocentral region (Fig. 2A) and rhythmic slow waves seen with the right frontal electrodes (Fig. 2B), especially during sleep.

The interictal neuropsychological examination showed moderate impairment of impulsivity, control behavior, disinhibition with frequent swearing, and impaired executive functioning with perseveration, compatible with a frontal lesion. Mild impairment of immediate recall and mild language deficits (paraphasia, lack of words) were also noted. Antiepileptic medication (barbexaclonum $100 \mathrm{mg} /$ day and diazepam $5 \mathrm{mg}$ /day) was started, reducing seizure frequency.

\section{Discussion}

The present case provides evidence of shared neural mechanisms for self-processing and vestibular processing in frontal cortex because both abnormal self-location and vestibular illusions occurred in this patient with right frontal lobe epilepsy. In addition to abnormal self-location, patients with OBEs also experience changes in their 1PP (elevated and dissociated from the body) and often see their own body (autoscopy) from this elevated location and perspective. But the patient described here did not experience abnormal $1 \mathrm{PP}$ and autoscopy.

A review of the literature by Blanke and Mohr [6] revealed that temporal lesions are observed in about $80 \%$, and parietal lesions in about $50 \%$, of patients with OBEs or heautoscopy, suggesting a prominent role of the TPJ in self-location and 1PP. There are only a few reports regarding the effects of frontal lesions on abnormal self-location and vertigo. Devinsky et al. [1] reported the case of a patient with sensations of floating, disembodiment, and autoscopy following the resection of a left frontotemporal arteriovenous malformation. In contrast, the abnormal self-location reported by our patient was associated with vestibular illusions, but not with autoscopy or abnormal 1PP. Neuroimaging data on frontal vestibular processing are also sparse and point to the anterior cingulum and the precentral, inferior, and middle frontal gyri (e.g., [7-9]). Kahane et al. [10] showed that electrical stimulation of the right inferior frontal gyrus may evoke sensations of body oscillations and disequilibrium, but without abnormalities in self-location. Hochman [11] described a patient with rotatory seizures caused
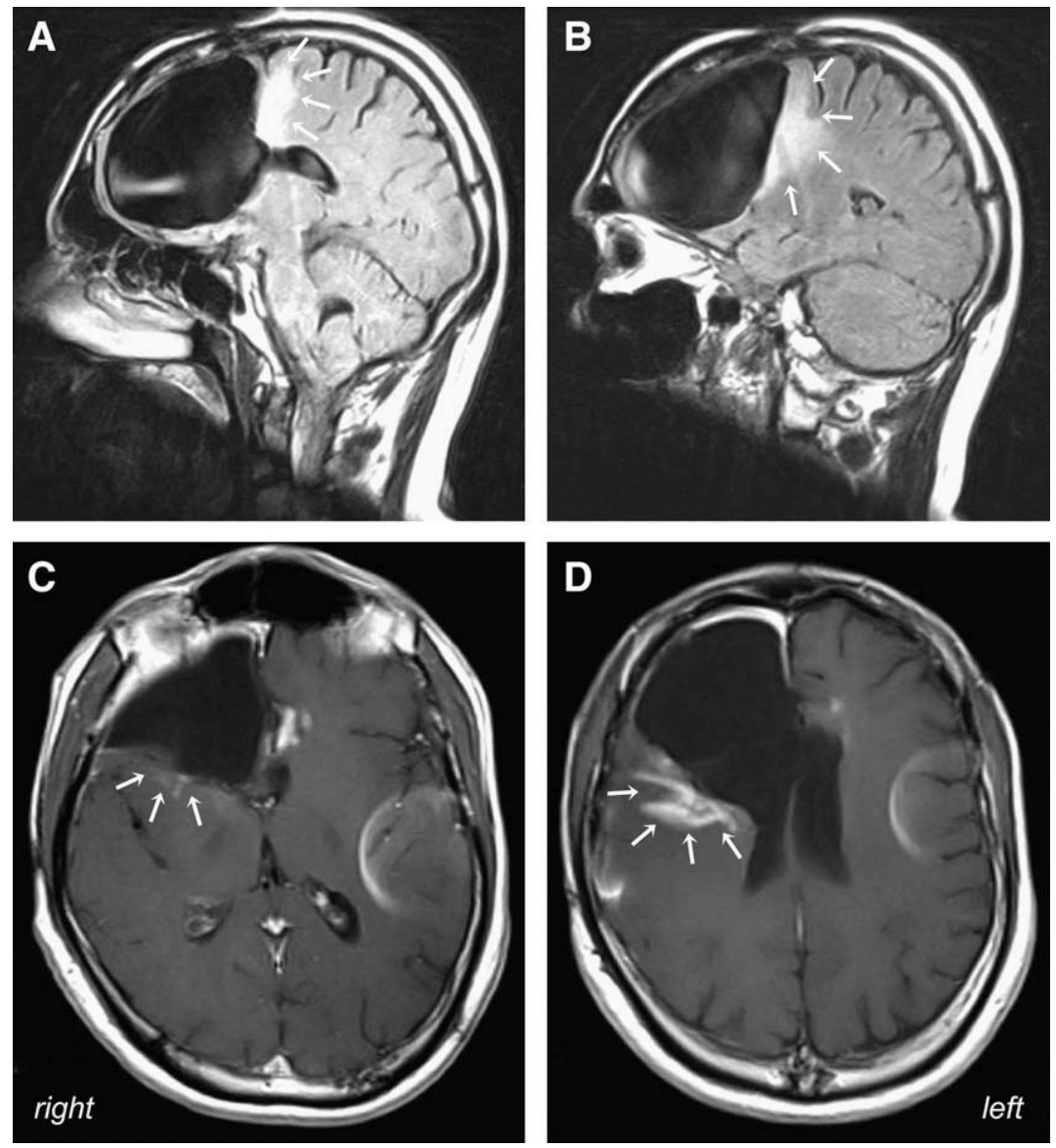

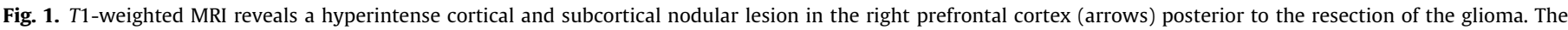
lesion enhances with contrast medium (gadolinium), indicating a relapse of the oligodendroglioma visible on the sagittal (A and B) and axial (C and D) planes. 
A

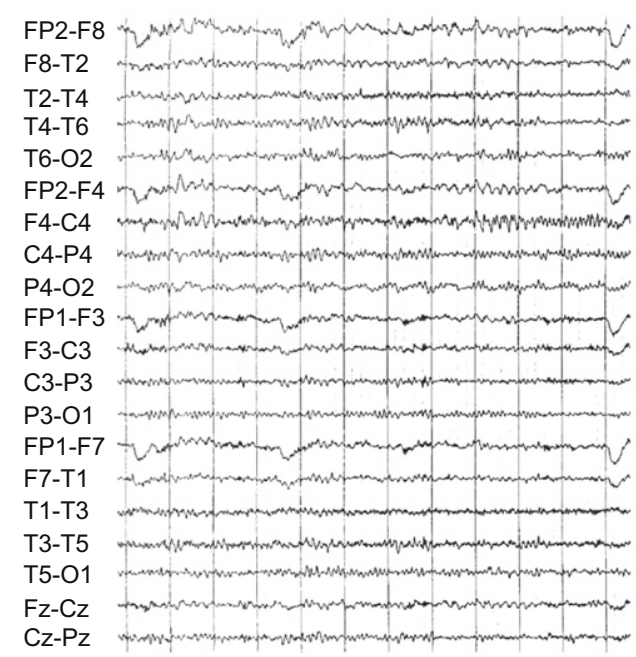

B

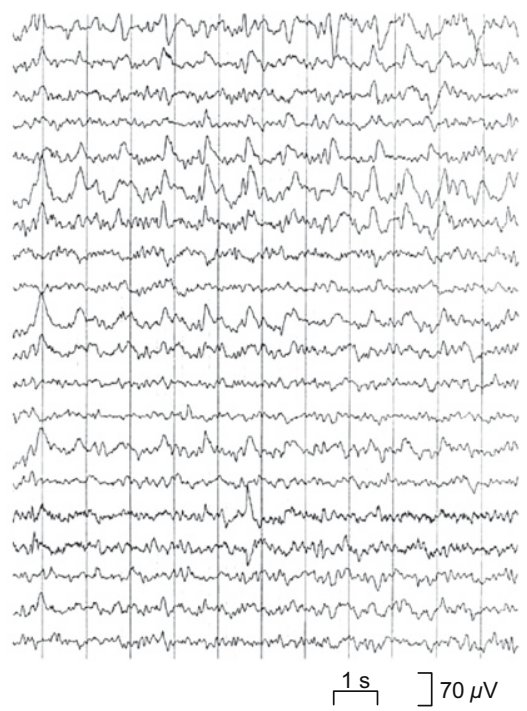

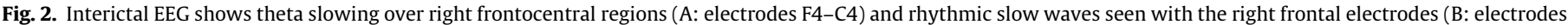
FP2-F4). A bipolar longitudinal montage is used.

by a right frontal neoplasm, with normal self-location and 1PP. Kluge et al. [12] described a young patient with an astrocytoma in the left middle frontal gyrus who experienced severe rotations of the visual surroundings (normal self-location and 1PP). Thus, despite some observations of vestibular sensations caused by frontal damage, the co-occurrence of vertigo and abnormal self-location has not been reported, or is rare, after frontal lesions, and these are not associated with autoscopy or change in 1PP. This is in contrast to the effects of damage at the TPJ, where abnormal self-location is often associated with abnormal 1PP and autoscopy.

The present observation therefore suggests that epileptic frontal lesions may induce multisensory disintegration and symptoms that differ from those occurring after TPJ damage. Regarding the multisensory mechanisms of abnormal self-location in our patient, we hypothesize that they are due mainly to a vestibularsomesthetic disintegration. First, our patient reported no associated changes in vision such as visual field changes, autoscopy, and 1PP, speaking against visuovestibular disintegration. Second, he had ictal somatosensory sensations characterized by an abnormal sensation of touch affecting his whole body. Because vestibular and somesthetic cues interact importantly to elaborate normal self-location, we therefore argue that the disintegration of vestibular and somesthetic cues in right precentral cortex led to both vertigo and disembodiment. A frontal contribution to self-location and self-related mechanisms gains further support from neuroimaging studies indicating abnormal frontotemporal connectivity in patients with personality disorders (e.g., [13]). Our data thus suggest that dysfunction of the frontal lobe, or of frontotemporal connectivity, may be associated with abnormal extracorporeal self-location. It should be noted that in the present case, epileptic activity may have spread to other adjacent frontal regions or to posterior regions involved in vestibular processing, for example, posterior insula, parietal or temporal cortex. Several frontal regions surrounding the lesion have been shown to be involved in vestibular processing, including premotor cortex [12], the frontal eye fields, the inferior frontal gyrus, precentral gyrus, and anterior cingulate cortex [8,9]. Posterior spread of ictal discharges may have involved the insula, temporal cortex, or parietal cortex [7-9,14]. For example, the posterior insula is thought to be the core region of the vestibular cortex, it integrates visual and somesthetic signals, and it is strongly intercon- nected with numerous other vestibular regions, including frontal cortex [15]. A posterior spread of the discharge to the insula is compatible with the appearance of ictal rotatory vertigo and loss of balance [16]. An additional contribution of the basal ganglia, close to the lesion location, cannot be excluded because they have been involved in rotational seizures caused by frontotemporal epilepsy $[17,18]$. The preserved $1 \mathrm{PP}$ may be due to the preserved integration of visuospatial with bodily signals, in contrast to what is observed during full-blown OBEs for which visual-vestibular-somesthetic disintegration seems to be necessary [3,6]. The absence of autoscopy in the present patient is also in line with literature indicating that autoscopy is associated with occipitotemporal lesions [6]. It should be noted that the experience of extracorporeal self-location without autoscopy, as well as other forms of bodily detachment, has been described in patients with epilepsy [1] and the healthy population. We propose that, because of the different patterns of multisensory disintegration (and different anatomical lesion sites), there is likely a continuum of altered bodily self-consciousness including fullblown OBEs (with abnormal self-location, 1PP, and autoscopy), incomplete OBEs (with abnormal 1PP and autoscopy, but normal self-location), abnormal self-location without autoscopy and normal 1PP (as in the present case), and autoscopy without changes in self-location and 1PP. Further clinical work is needed merging analysis of symptoms and functional and neural mechanisms of bodily self-consciousness. This seems necessary to provide patients with scientific explanations for their complex seizure manifestations and because of the methodological difficulties involved in testing these mechanisms experimentally in healthy subjects.

\section{Acknowledgment}

This research was supported by the Swiss National Science Foundation (SINERGIA CRSII1-125135/1; SNF 320030-122073; SNF 320030-113766 and SNF 33CM30-124089).

\section{References}

[1] Devinsky O, Feldmann E, Burrowes K, Bromfield E. Autoscopic phenomena with seizures. Arch Neurol 1989;46:1080-8. 
[2] Brugger P. Illusory reduplication of one's own body: phenomenology and classification of autoscopic phenomena. Cogn Neuropsychiatry 1997;2:19-38

[3] Blanke O, Landis T, Spinelli L, Seeck M. Out-of-body experience and autoscopy of neurological origin. Brain 2004;127:243-58.

[4] Podoll K, Robinson D. Out-of-body experiences and related phenomena in migraine art. Cephalalgia 1999;19:886-96.

[5] Maillard L, Vignal JP, Anxionnat R, TaillandierVespignani L. Semiologic value of ictal autoscopy. Epilepsia 2004;45:391-4

[6] Blanke O, Mohr C. Out-of-body experience, heautoscopy, and autoscopic hallucination of neurological origin: implications for neurocognitive mechanisms of corporeal awareness and self-consciousness. Brain Res Brain Res Rev 2005;50:184-99.

[7] Bottini G, Karnath HO, Vallar G, et al. Cerebral representations for egocentric space: functional-anatomical evidence from caloric vestibular stimulation and neck vibration. Brain 2001;124:1182-96.

[8] Bense S, Stephan T, Yousry TA, Brandt T, Dieterich M. Multisensory cortical signal increases and decreases during vestibular galvanic stimulation (fMRI). J Neurophysiol 2001;85:886-99.

[9] Lobel E, Kleine JF, Le Bihan D, Leroy-Willig A, Berthoz A. Functional MRI of galvanic vestibular stimulation. J Neurophysiol 1998;80:2699-709.
[10] Kahane P, Hoffmann D, Minotti L, Berthoz A. Reappraisal of the human vestibular cortex by cortical electrical stimulation study. Ann Neurol 2003;54:615-24.

[11] Hochman MS. Rotatory seizures associated with frontal lobe malignant neoplasm: a case report. Epilepsia 1983;24:11-4.

[12] Kluge M, Beyenburg S, Fernandez G, Elger CE. Epileptic vertigo: evidence for vestibular representation in human frontal cortex. Neurology 2000;55:1906-8.

[13] Nakamura M, McCarley RW, Kubicki M, et al. Fronto-temporal disconnectivity in schizotypal personality disorder: a diffusion tensor imaging study. Biol Psychiatry 2005;58:468-78.

[14] Blanke O, Perrig S, Thut G, Landis T, Seeck M. Simple and complex vestibular responses induced by electrical cortical stimulation of the parietal cortex in humans. J Neurol Neurosurg Psychiatry 2000;69:553-6.

[15] Guldin WO, Grüsser OJ. Is there a vestibular cortex? Trends Neurosc $1998 ; 21: 254-9$

[16] Brandt T, Botzel K, Yousry T, Dieterich M, Schulze S. Rotational vertigo in embolic stroke of the vestibular and auditory cortices. Neurology 1995;45:42-4.

[17] Vercueil L, Kahane P, Francois-Joubert A, et al. Basal ganglia involvement in rotational seizures. Epileptic Disord 1999;1:107-12.

[18] Vercueil L, Hirsch E. Seizures and the basal ganglia: a review of the clinical data. Epileptic Disord 2002;4(Suppl. 3):S47-54. 